Juergen Kratzsch*, Mandy Vogel, Tanja Poulain and Wieland Kiess

\title{
New reference intervals for endocrinological biomarkers in pediatric patients: what can we learn from the LIFE child study?
}

https://doi.org/10.1515/labmed-2021-0145

Received October 10, 2021; accepted October 24, 2021; published online November 11, 2021

\section{Abstract}

Objectives: We established reference intervals for serum concentrations of hormones from healthy pediatric subjects and investigated their associations with gender, body mass index (BMI), puberty and oral contraceptives (oC).

Methods: We calculated reference intervals for the thyroid parameters thyroid-stimulating hormone (TSH), free triiodothyronine (FT3), free thyroxine (FT4), and calcitonin (Ct); the bone markers osteocalcin, procolagen type $1 \mathrm{~N}$-propeptide, and carboxy-terminal cross-linking telopeptide of type 1 collagen; the calciotropic hormones 25-hydroxyvitamin D (25(OH)D) and parathyroid hormone and the steroids cortisol, progesterone, 17-hydroxyprogesterone, androstenedione, testosterone, estradiol, dehydroepiandrosterone sulfate and aldosterone. Up to 10,002 blood serum samples from 3,229 healthy children and adolescents (age interval: 3 months to 20 years) were measured. To investigate the associations between the hormone levels with age, sex, weight status and the role of puberty-based changes, the measurement and BMI values were transformed into standard deviation scores.

Results: Most of the hormones depended on age- and gender. Puberty was linked to a, in part, temporary decrease in TSH, FT3 (for females), FT4, Ct, cortisol (for girls) and aldosterone (for boys) and peak in the bone marker and calciotropic hormones (excluding 25(OH)D) and nearly all remaining steroids. BMI had effects on the

\footnotetext{
*Corresponding author: Prof. Dr. rer. nat. Juergen Kratzsch, Institute of Laboratory Medicine, Clinical Chemistry and Molecular Diagnostics, University Hospital Leipzig, Paul-List-Str. 13-15, 04103 Leipzig,

Germany, Phone: +01739044771,

E-mail: juergen.kratzsch@medizin.uni-leipzig.de

Mandy Vogel and Tanja Poulain, LIFE Leipzig Research Center for Civilization Diseases, Leipzig University, Leipzig, Germany Wieland Kiess, LIFE Leipzig Research Center for Civilization Diseases, Leipzig University, Leipzig, Germany; and Department of Women and Child Health, University Hospital for Children and Adolescents and Center for Pediatric Research, Leipzig University, Leipzig, Germany
}

thyroid, bone, and calciotropic parameters, whereas oC led to increased cortisol, suppressed progesterone and estradiol values.

Conclusions: Age- and gender-specific reference intervals are essential for the interpretation of pediatric patients' hormone measurements. Influencing factors as puberty, BMI, or oC should be taken into consideration for diagnosis and treatment monitoring.

Keywords: bone marker; calcitonin; confounder; reference interval; steroid; thyroid.

\section{Introduction}

Clinically relevant reference intervals are a mandatory precondition for the interpretation of endocrine biomarker measurements. The absolute level of the biomarker is determined by the basal stage and functional capacity of the hormone-producing cells. Additionally, various confounders may regulate the final circulating level of each hormone. In pediatric patients, age and sex are the most important covariates because both determine growth and maturity during childhood. At the onset of adolescence, puberty-induced sex hormones can be observed and may stimulate the hormone levels of the related endocrine axis (e.g., the bone turnover marker). Although pubertal development can be determined by the individual Tanner stage, the role of puberty as an endocrine confounder for reference values remains largely ambiguous. This is mainly caused by the issue that patients, physicians or study assistants frequently refuse the examination of pubertal features of adolescents.

Recently, overweight or obesity has been found to be prevalent in every sixth child and tends to persist into later life [1]. An increase in the amount of adipose tissue may have effects on growth and maturity during childhood, but also on the pubertal development of children and adolescents. This can be observed, for instance, as increasing levels of the sex hormones testosterone and estradiol from Tanner Stages $1-5$ or by the increase of dehydroepiandrosterone sulfate over the adrenarche. However, it remains unclear whether or not the secretion of hormones 
from another axis (e.g., bone markers or thyroid hormones) is under the control of BMI and puberty.

The LIFE Child study (clinical trial number NCT02550236) is a population-based longitudinal cohort study conducted at the Research Center for Civilization Diseases in Leipzig. It aims to monitor healthy child development from birth to adulthood and to understand the etiology of lifestyle diseases such as obesity [2]. The study consists of three interrelated cohorts; the birth cohort, the health cohort, and the obesity cohort (Figure 1). Subjects were invited to participate at 3, 6 and 12 months of age, followed by annual invitations from 1 to 18 years of age. Due to the large numbers of healthy infants, children and adolescents included in the study, the use of their laboratory and clinical data to establish reference values for endocrine biomarkers was a temptation for each clinical chemist. Moreover, the LIFE study investigated various confounders in detail, such as puberty, weight status, medication, and others.

Thereby we were able to assess whether confounders such as puberty, body mass index (BMI), and the frequently neglected use of contraceptives have effects on age- and gender-specific reference intervals for the thyroid parameters thyroid-stimulating hormone (TSH), free triiodothyronine (FT3), free thyroxine (FT4), and calcitonin (Ct); the bone markers osteocalcin, procollagen type $1 \mathrm{~N}$-propeptides (PINP) and carboxy-terminal cross-linking telopeptide of type 1 collagen (CTX-I), the calciotropic hormones 25-hydroxyvitamin D (25(OH)D) and parathyroid hormone (PTH); as well as for the steroids cortisol, progesterone, 17-hydroxyprogesterone, androstenedione, testosterone, estradiol, dehydroepiandrosterone sulfate, and aldosterone. Accordingly, this article presents a brief overview from the current set of four published articles on endocrine biomarkers from the LIFE Child study [3-6].

\section{Materials and methods}

\author{
Laboratory assessment
}

Venous blood was taken in the morning between 7:30 and 10:00 from $90 \%$ of subjects who had fasted overnight, $96 \%$ by $11: 00,99 \%$ by $12: 00$ and $100 \%$ by $16: 00$. Samples were processed by trained staff at the LIFE Biobank following standard operating procedures and sent directly to the Institute of Laboratory Medicine, Clinical Chemistry and Molecular Diagnostic at the University Hospital Leipzig.

Steroid hormones in serum were simultaneously quantified using liquid chromatography-tandem mass spectrometry (LC-MS/MS) after solid phase extraction. Steroids can be analyzed in $4 \mathrm{~min}$ after a single manual dilution and protein precipitation step. Method specifications were recently described in detail in refs. [7, 8]. The remaining biomarkers were measured by electrochemiluminescence assays (ECLIA) via Cobas ${ }^{\circledR} 601$ or 801 (Roche Diagnostics GmbH, Germany).

\section{Quality control data}

Thyroid parameter: The mean inter-assay coefficient of variation of the four measured biomarkers ranged from SD 2.25-3.11\%, and the mean deviation from the target value was between 3.33 and $4.82 \%[3,4]$. Bone and calcium homeostasis parameter: The mean inter-assay coefficient of variation of the five measured bone biomarkers ranged from 1.95 to $13.10 \%(25(\mathrm{OH}) \mathrm{D})$ from four quality control cycles over representative four months ( $\mathrm{n}=82-164$ runs) [5]. Steroid hormones: The mean inter-assay coefficient of variation of the nine measured bone biomarkers ranged from 2.9 to $14.8 \%$ (DHEAS) for $n=58$ runs [6].

\section{Subjects}

For TSH and the thyroid hormones, the final cohort comprised 3,140 children and adolescents, from whom 9,404 serum samples were measured [3]. Inclusion criteria were the availability of age, gender and basic anthropometric and medical history information. Subjects who suffered from thyroid function-affecting diseases were excluded.

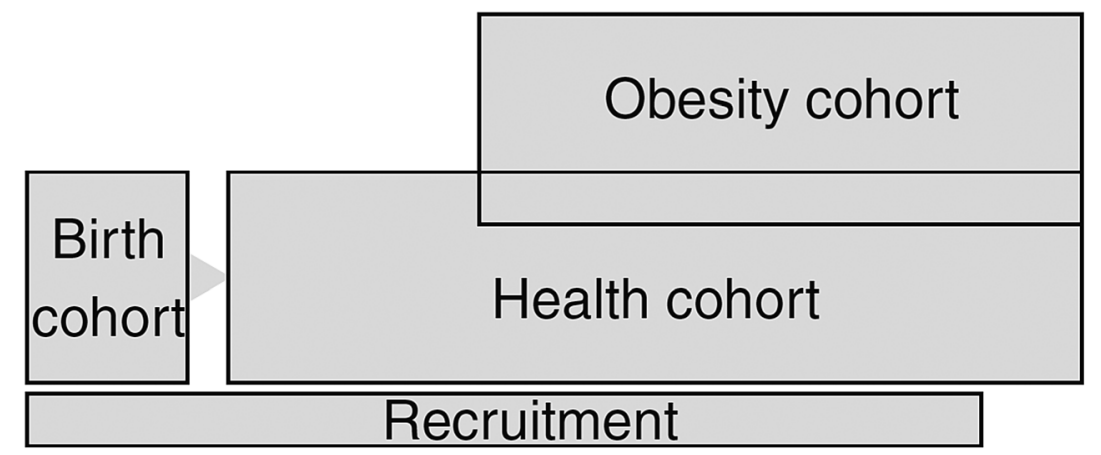

24W 36W 03M 06M 01Y 02Y 03Y 04Y 05Y 06Y 07Y 08Y 09Y $10 \mathrm{Y} 11 \mathrm{Y} 12 \mathrm{Y} 13 \mathrm{Y}$ 14Y $15 \mathrm{Y}$ 16Y $17 \mathrm{Y} 18 \mathrm{Y}+$ Time point
Figure 1: Study cohorts in the LIFE Child study and their interrelations. $W$, weeks of gestation; $M$, age of children in month; $\mathrm{Y}$, age of children in years. With permission from ref. [2]. 
Weight groups were defined as follows: underweight as a BMI lower than the 10th percentile (SDS $<-1.281$ ), normal weight between the 10th and 90th percentiles (SDS -1.281 to 1.282) and overweight as a BMI higher than the 90th percentile (SDS>1.282) in line with the German reference data according to current national guidelines [9].

For the Ct reference intervals, the exclusion criteria were endocrine disorders, coagulation disorders, mental illnesses, or diseases of bone, muscles, kidney, liver, the cardiovascular system, or the gastrointestinal tract [4]. Subjects taking medication that could interfere with hormones or bone metabolism were also excluded. As a result, the study cohort consisted of 2,636 subjects between the ages of approximately 2 months and 18 years (1,236 females and 1,400 males) with 5,410 measurements (2,486 females and 2,924 males).

For bone and calciotropic parameter sera, 3,183 subjects with 7,740 visits were included (see ref. [5]). Subjects with height or weight standard deviation scores (SDS) $<-5$ or $>5$, chronic disease (e.g., endocrine disorders, metabolic disorders, diabetes mellitus, chromosomal disorders, chronic kidney and liver diseases), or taking medications (e.g., glucocorticoids, metformin, l-thyroxin) that could affect bone metabolism were excluded (1,402 serum samples from 492 subjects). Moreover, we excluded extremely underweight subjects (BMI-SDS<-1.881, 135 serum samples from 31 subjects) and obese subjects (BMI-SDS >1.881). To assess the effects of BMI on bone markers and calciotropic hormones, we included all obese individuals with BMI-SDS >1.881 as a separate sub-cohort ( 489 blood samples from 317 subjects, 1-6 serum samples per subject).

For steroids, a total of 4,678 serum samples from healthy volunteers were analyzed (age: 0.33-79 years, 2,512 males, 2,166 females) within the scope of two population-based LIFE-cohorts [6]. Subjects with concomitant endocrine diseases, glucocorticoid therapy, hepatitis, pregnancy, or body mass index (BMI) $\geq 33$ in adults or BMI standard deviation score (SDS) below -3 or over +3 in children and adolescents were excluded.

\section{Anthropometric measures and pubertal status}

Certified and trained study assistants from the LIFE Child study center measured anthropometric data and performed regular plausibility checks.
Tanner stages (TS, age $>5$ years, $[10,11])$ representing the pubertal status of adolescents were determined by certified same-gender staff members.

\section{Statistics}

The measured values of the biomarkers were transformed into standard deviation scores (SDS), and their associations with age, gender, and puberty were analyzed. Body mass index (BMI) was transformed into SDS by applying German reference data according to current national guidelines [9]. Age- and gender-specific reference intervals were derived from a reference cohort consisting of normal weight children by applying an LMS-type method as implemented in the gamlss package (for details see ref. [12]). To avoid a violation of the independence assumption, $75 \%$ of the families were randomly selected, and from those, in turn, one measurement was randomly chosen. Reference intervals were estimated from this sub-sample. The procedure was repeated 1,000 times to determine the average estimated values and their confidence limits. It allows all measured data to be included. The 2.5th, 50.0th (median) and 97.5th percentiles were visually represented.

\section{Results}

The gender-specific age-dependent reference values derived from our studies are presented for the thyroid parameters in Figure 2; for $\mathrm{Ct}$ in Figure 3, for the bone marker and calcium homeostasis parameters in Figure 4 and for steroids in Figure 5.

Moreover, details on the effects of age, gender, puberty, BMI-SDS and oC on the reference values are summarized in Table 1. The most important results are described below:

- TSH and FT3 demonstrated a decrease in hormone levels before puberty, whereas the age-dependent
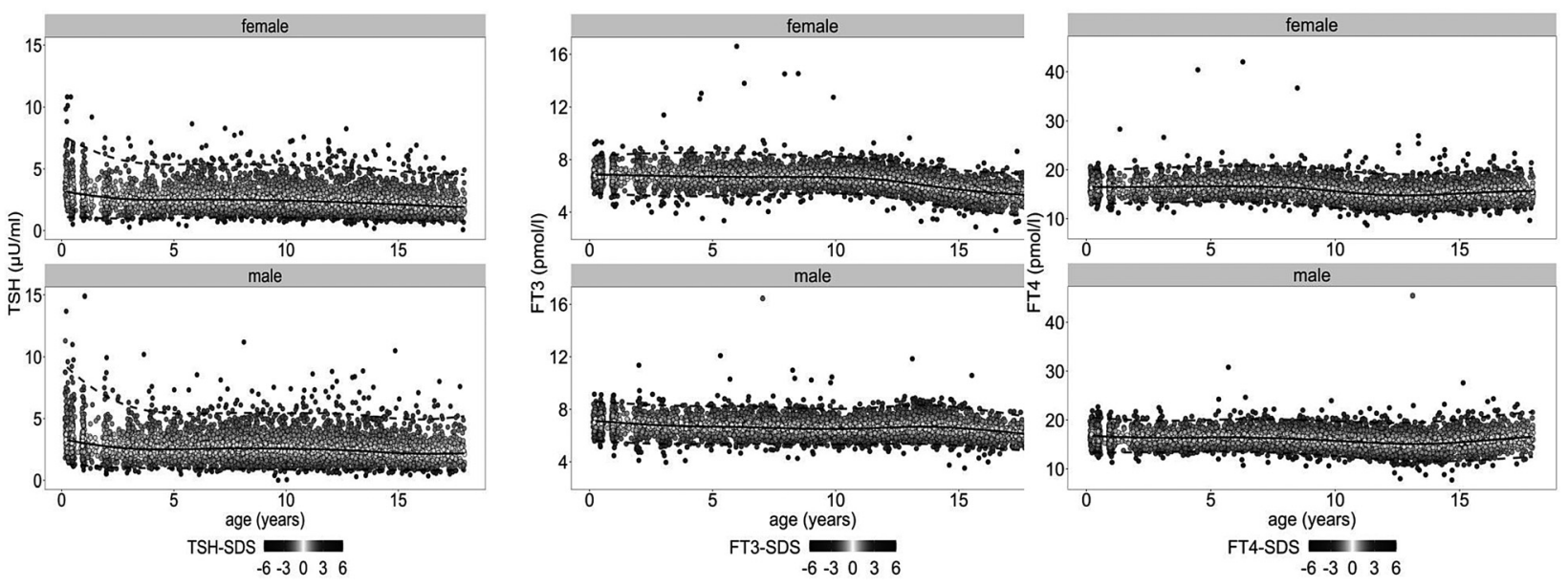

Figure 2: Reference percentiles for TSH, FT3, and FT4 by age and sex.

The 2.5th (dashed line), 50th (solid line), and 97.5th (dashed line) percentiles for males and females are shown for TSH (A), FT3 (B), and FT4 (C). The intensity of the shading of the hormone level data points for the reference cohort indicates the relative hormone SDS value. SDS, standard deviation score. With permission from ref. [3]. 

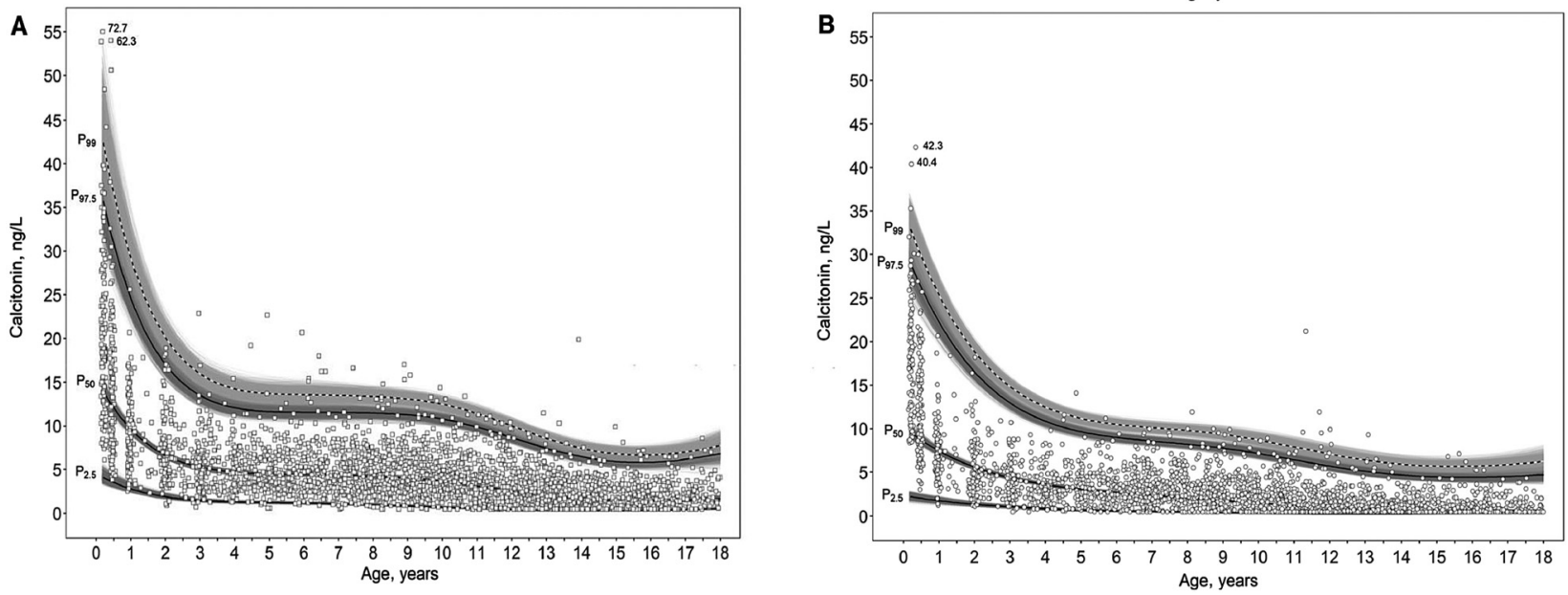

Figure 3: Serum Ct measurements of healthy boys (A) and girls (B) depending on age.

A solid line indicates the 2.5 th, 50th, and 97.5 th percentiles. The 99 th percentile was shown as a dashed line. Re-sampling levels were marked by the shaded areas around the percentiles. With permission from ref. [4].

changes in FT4 were rather negligible (Figure 2). Gender-dependent small variations were observed for a restricted age range in TSH and FT3. During puberty, TSH and FT3 were lowest during P5 and P3, respectively. FT4 was increased at P3 or P4. TSH-SDS and FT3-SDS demonstrated a significant positive and FT4-SDS a significant negative correlation with BMI-SDS, leading to distinctly different thyroid values in obese subjects [3].

- Ct showed an age-dependent decrease, especially in infants (Figure 3). Puberty or high BMI-SDS in children older than six years were associated with slightly reduced values [13].

- Osteocalcin, PINP and CTX-I showed a distinct ageand gender-dependence with peak levels at 10-11 years (girls, Tanner 3) and 13 years (boys, Tanner 3-4) (Figure 4). For 25(OH)D and PTH, we found no distinct age-dependency. The maximum of 25(OH)D in infants is likely to be induced by exogenous supplementation. During puberty, the values of osteocalcin, P1NP and CTX-I reached their maximum later in boys than in girls. A faint peak was also detected in $\mathrm{P} 3$ or $\mathrm{P} 4$ for $\mathrm{PTH}$ and there was a respective nadir for 25(OH)D in $\mathrm{P} 4$. Interestingly, the mean SDS levels in our obese cohort differed significantly $(p<0.001)$ from the values in the reference cohort: Osteocalcin by -0.44 , PINP by -0.27 , CTX-I by $-0.33,25(\mathrm{OH}) \mathrm{D}$ by -0.43 , PTH by +0.44 [5].

- Excluding cortisol and aldosterone, all other steroid hormones showed at least slightly increased serum values during mini-puberty in the age interval of around 0.5-1 year (Figure 5; Ref. [6]). High aldosterone levels during the same period revealed physiological hyperaldosteronism due to renal tubular immaturity and mineralocorticoid insensitivity. A similar trend could be seen during the actual puberty, whereas cortisol appeared to demonstrate slightly increasing values. We also found the well-known difference between boys and girls with higher levels of male testosterone and female estradiol and progesterone, at least with puberty onset. Interestingly, distinctly increased cortisol and to a lower extent testosterone levels were measured when adolescent girls used oC. Unfortunately, this effect was not investigated in the non-steroid hormone studies.

\section{Discussion}

Adult reference intervals for hormones are frequently presented by the manufacturer's package insert as applicable for routine diagnostics of endocrinological diseases in infants, children or adolescents. However, if adult reference intervals are applied in pediatrics, the number of diagnostic errors will be distinctly higher due to differences in the physiology of both groups, especially the hormone secretion. Our study found that covariates such as age and gender as well as confounders such as puberty, BMI, or contraceptive use were responsible for significant variations in the reference intervals of all 17 investigated hormone biomarkers during infancy, childhood and adolescence. Although these variations are important for the clinical interpretation for most of the measures, single parameters (e.g., FT4) demonstrated 

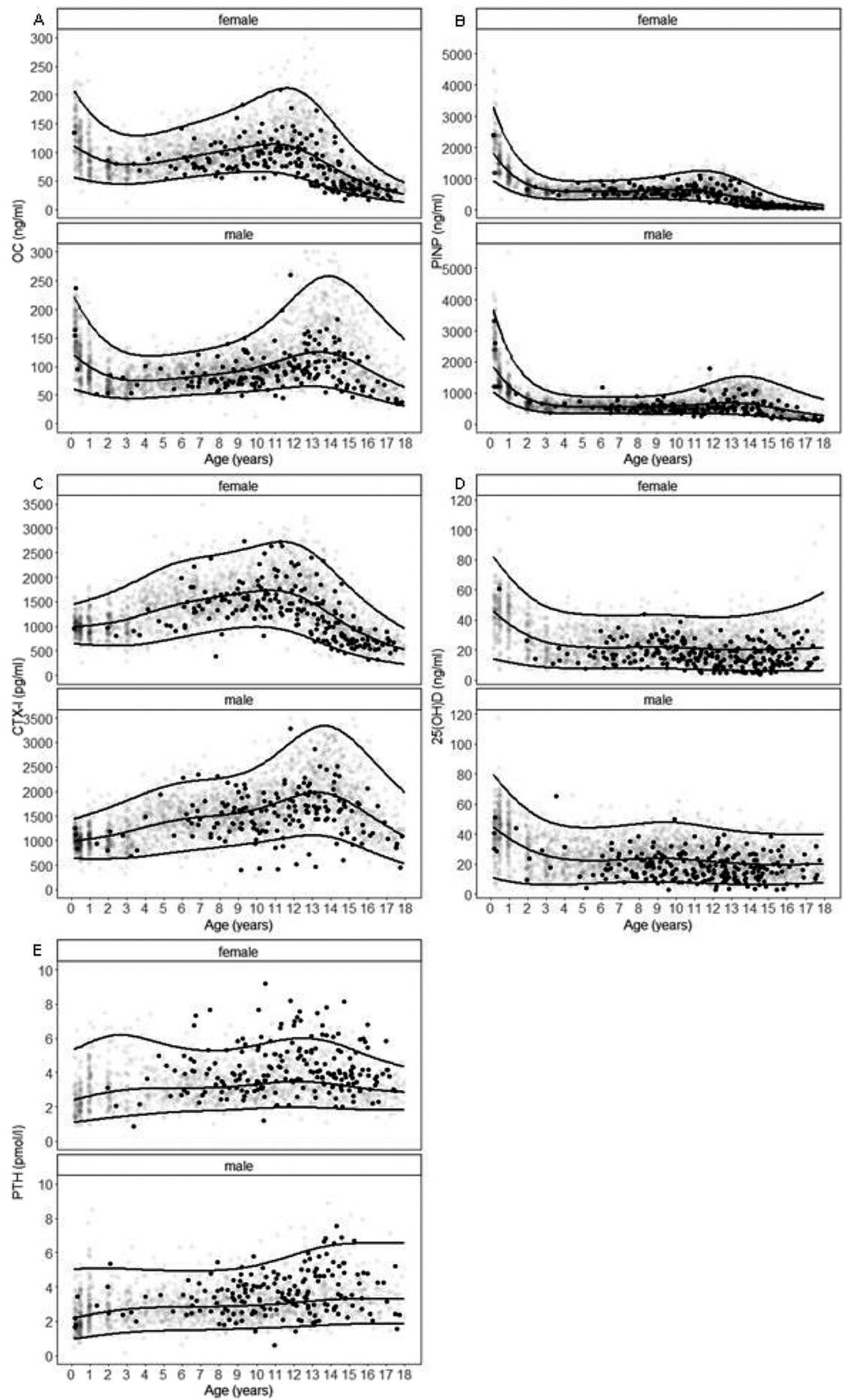

Figure 4: Percentiles for osteocalcin (OC), PINP, CTX-I, 25(OH)D, and PTH by age and gender.

Boys' and girls' percentiles are shown (2.5th, 50th, 97.5th) for the OC (A), PINP (B), 459 CTX-I (C), 25(OH)D (D), and PTH

(E) levels in the reference cohort (transparent dots), and the distribution of levels for obese children (BMI-SDS >1.88) are shown as black dots. With permission from ref. [5].

only minor covariate- and confounder-related changes that are largely negligible for clinical practice.

We observed age-related changes in reference intervals between birth and puberty. The physiological post-partum maturation was related to an adjusted secretion of hormones, especially in bone-, growth- and energy-related hormone axes. Moreover, minipuberty [14] is characterized by an increase in sexual hormones with huge inter-individual variations and has to be considered while interpreting diagnostic or therapeutic findings.

During puberty, gender is the major influencing factor, especially as the individual stages of puberty are delayed in boys compared to girls. The stages themselves represent the different puberty-related secretion capacity of sex hormones and are, therefore, the most important confounder of hormones during adolescence. Accordingly, we found respective 
Cortisol (nmol/L)

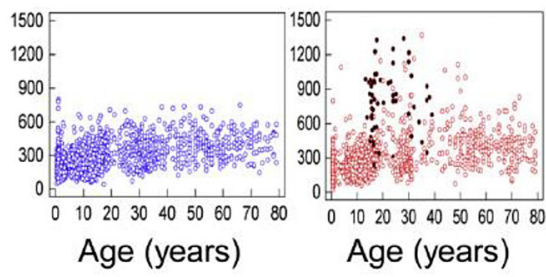

Age (years) Age (years)

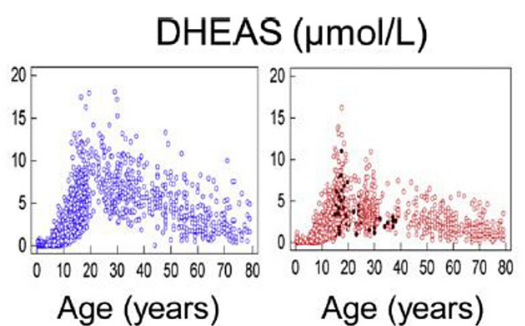

Age (years)

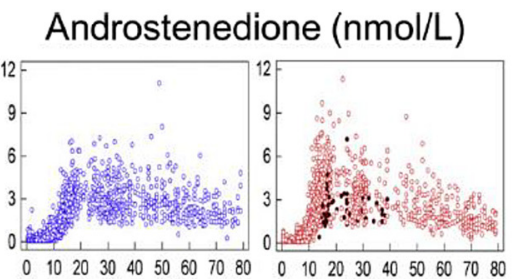

Age (years)
Age (years)
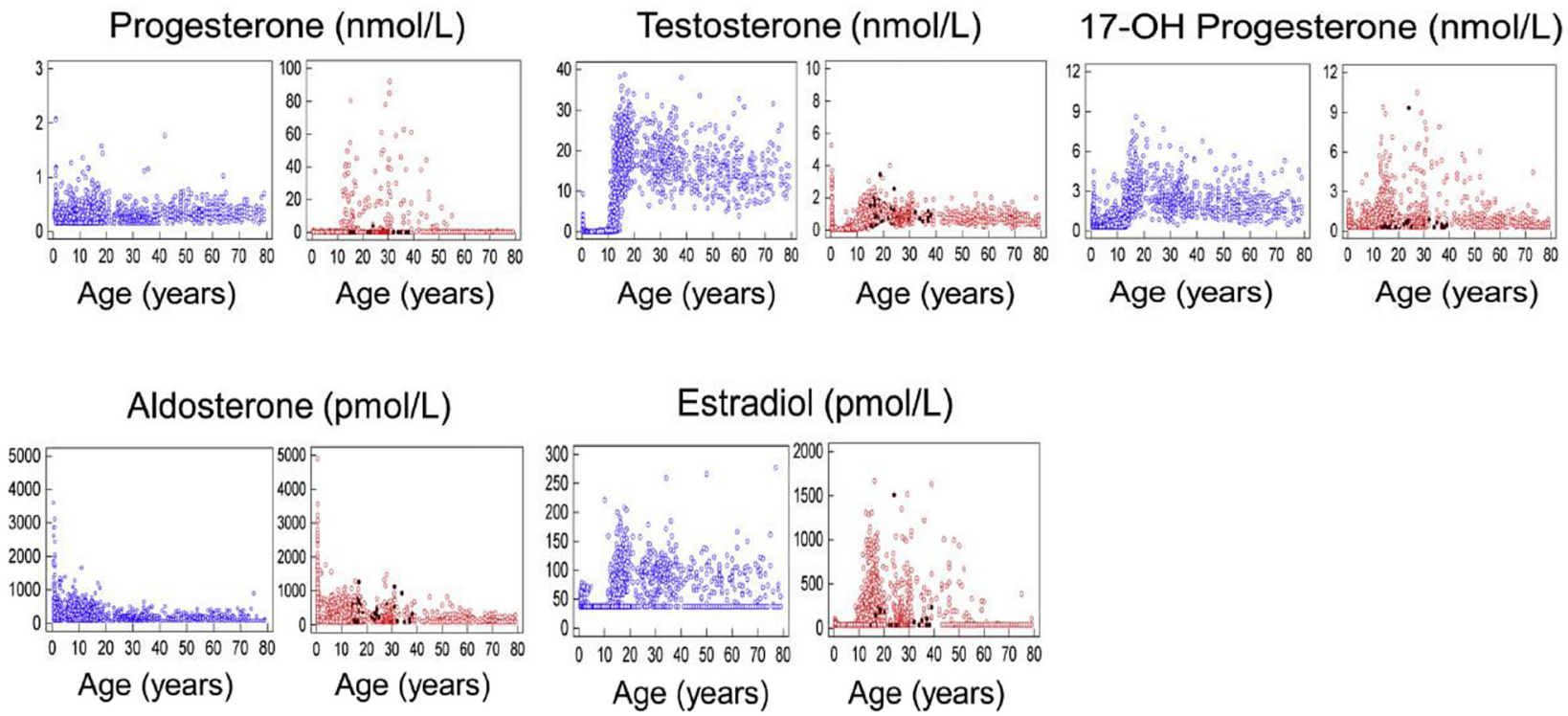

Figure 5: Steroid hormone levels change throughout the lifespan in males and females.

Blue: males; red: females without oral contraceptives (oC); black: females with oC. With permission from ref. [6].

changes in hormone levels for 15 of our 17 parameters, although some of them were rather small. Moreover, other hormones, such as the biomarkers of the growth-hormone insulin-like growth factor (IGF) axis, IGF-I [15] and IGF binding protein-3 [16], showed a corresponding peak in their serum levels during puberty.

Interestingly, we were able to demonstrate an effect of BMI on thyroid function, bone markers and the calciotropic hormones 25(OH)D and PTH. The relationship between BMI and TSH was recently suggested by an Israeli group by a data mining approach [17]. Accordingly, in obese children, conspicuous findings regarding these two axes have to be interpreted with caution. This is also true for cortisol and testosterone in girls taking oC: the drug-induced increased binding proteins for cortisol and testosterone led to dramatically increased values for both steroids.

The outstanding unique feature of our study is the huge sample of primarily healthy subjects whose ages covered the entire interval of infancy (excluding the neonatal period), childhood and adolescence. All of them were examined at a single study center, following the same highly standardized methods carried out by professionally trained staff. In the literature, such so-called "direct" procedures for establishing reference intervals have been very rare due to the large effort and costs. To the best of our best knowledge, there was only one analogous approach known as the "Canadian Laboratory Initiative on Pediatric Reference Intervals" (CALIPER) [18], which recruited subjects from across Canada, but was not primarily focused on endocrine parameters. Due to the large number of included subjects, LIFE Child and CALIPER were able to detect statistically significant moderate or even small confounding effects on the reference intervals. Moreover, there was no need to integrate samples or data from different clinical studies obtained from different centers located in different international regions, an approach that frequently fails in quality control or in collecting detailed, reliable and consistent data from all patients.

Recently, "indirect" procedures have been applied more and more often to establish pediatric reference intervals (e.g. data mining) in lieu of extensive and laborious "direct" reference interval studies. However, such 
Table 1: Overview of the effect of age before puberty, gender, puberty, body mass index standard deviation score (BMI-SDS), and the use of contraceptives on reference intervals for 17 hormones.

\begin{tabular}{|c|c|c|c|c|c|c|}
\hline Parameters & Method [Ref.] & $\begin{array}{l}\text { Change with age } \\
\text { before pub start }\end{array}$ & Gender & Pub & $\begin{array}{l}\text { Correlation BMI-SDS vs. } \\
\text { hormone-SDS }\end{array}$ & Oral contraceptives \\
\hline TSH & $\mathrm{IA}[2]$ & Infants $\downarrow$, later $\downarrow$ & $\begin{array}{l}m>f(1-4, \\
13-15 \text { years })\end{array}$ & Min P5 & Positive & N.a. \\
\hline FT3 & IA [2] & $\downarrow$ & $\begin{array}{l}m<f(9-12 \text { years }) \\
m>f(12-18 \\
\text { years })\end{array}$ & Max P3 or P4 & Positive & N.a. \\
\hline FT4 & $\mathrm{IA}[2]$ & N.d. & $m \sim f$ & Min P3 & Negative & N.a. \\
\hline Calcitonin & IA [3] & Infants $\downarrow \downarrow$, later $\downarrow$ & $m>f$ & $\downarrow$ & Minor negative & N.a. \\
\hline Osteocalcin & IA [4] & Infants $\downarrow$ & m pub later & $\uparrow \uparrow \uparrow$ & Negative & N.a. \\
\hline PINP & IA [4] & Infants $\downarrow$ & $\mathrm{m}$ pub later & $\uparrow \uparrow$ & Negative & N.a. \\
\hline CTX-I & IA [4] & Infants $\uparrow$ & $\mathrm{m}$ pub later & $\uparrow \uparrow \uparrow$ & Negative & N.a. \\
\hline $25(\mathrm{OH}) \mathrm{D}$ & IA [4] & Unclear (substitution?) & $m \sim f$ & N.d. & Negative & N.a. \\
\hline PTH & IA [4] & N.d. & $m \sim f$ & $\uparrow$ & Positive & N.a. \\
\hline Cortisol & LC-MS/MS [5] & N.d. & $m \sim f$ & $\uparrow$ & N.a. & $\uparrow \uparrow \uparrow$ \\
\hline Progesterone & LC-MS/MS [5] & Minipub $\uparrow$, later? & $f>\gg>m$ after pub & $\uparrow \uparrow \uparrow$ & N.a. & N.d. \\
\hline $17-\mathrm{OH}$ progesterone & LC-MS/MS [5] & Minipub $\uparrow$, later? & $m \sim f$ & $\uparrow \uparrow \uparrow$ & N.a. & N.d. \\
\hline Androstendione & LC-MS/MS [5] & Minipub $\uparrow$, later? & $m \sim f$ & $\uparrow \uparrow \uparrow$ & N.a. & N.d. \\
\hline Testosterone & LC-MS/MS [5] & Minipub $\uparrow$, later? & $m \gg \gg>f$ after pub & $\uparrow \uparrow \uparrow$ & N.a. & $\uparrow$ \\
\hline Estradiol & LC-MS/MS [5] & Minpub $\uparrow$, later? & f $\gg>m$ after pub & $\uparrow \uparrow \uparrow$ & N.a. & N.d. \\
\hline DHEAS & LC-MS/MS [5] & Minipub $\uparrow$, later $\uparrow$ & $m \sim f$ & $\uparrow \uparrow \uparrow$ & N.a. & N.d. \\
\hline Aldosterone & LC-MS/MS [5] & $\downarrow$ & $m \sim f$ & N.a. & N.a. & N.d. \\
\hline
\end{tabular}

TSH, thyroid stimulating hormone; FT3, free triiodothyronine; FT4, free thyroxine; PINP, procollagen type 1 N-propeptides; CTX-I, carboxyterminal cross-linking telopeptide of type 1 collagen; 25(OH)D, 25 hydroxy vitamin D; PTH, parathyroid hormone; DHEAS, dehydroepiandrosterone-sulfate; IA, immunoassay; LC-MS-MS, liquid chromatography coupled double mass spectrometry; references [Ref.] are presented in square brackets; N.d., not detected; pub, puberty; $\downarrow$, decreasing hormone level; $\uparrow$, increasing hormone level; ?, unclear; m, males; f, females; min, minimum; max, maximum; P, puberty stage according to Tanner; est., estimated; N.a., not available; contracept., contraceptives.

"indirect" procedures need routine findings from huge numbers of patients released from the laboratory archive. Thus, this procedure is usually applied for parameters from classical clinical chemistry and blood cell analysis [19, 20]. Publications of validated hormone reference intervals based on data mining procedures are largely restricted to thyroid parameters [21]. More research is needed to develop a respective approach covering the entire spectrum of hormones for diagnostics in pediatric endocrinology.

The reference intervals for the thyroid parameters, the bone markers, and parameters of calcium homeostasis were based on the measurement using an ECLIA method; for steroid measurement, we used the liquid chromatography tandem mass spectrometry (LC-MS/MS). The latter method was developed to improve the comparability of results from different analytical methods and to overcome potential interference from immunoassays [8]. However, it is still infrequently used in routine labs so far.

Strictly speaking, our reference intervals are valid only for a Middle-European, Caucasian pediatric population. However, qualitatively, the confounder-dependent course of our biomarkers should be considered and interpreted along with the patient's clinical appearance, independently on the used analytical method. Moreover, our reference intervals can be adapted for immunoassays of different manufacturers or for different LC-MS/MS approaches after mathematical transformation and respective validation according to CLSI guideline C28-A3 [22].

We conclude that age- and gender-specific reference intervals are important for the correct interpretation of hormone measurements in infants, children and adolescents. In addition, confounding factors such as puberty, BMI and the use of contraceptives should be considered when hormones are used for the diagnosis and treatment monitoring of endocrine diseases.

Acknowledgments: (Not applicable).

Research funding: The LIFE child study was financed by the excellence initiative of the Saxon State Ministry for Science and Art, Saxony, Germany and the European Regional Development Fund of the European Union.

Author contributions: All authors have accepted responsibility for the entire content of this manuscript and have approved its submission.

Competing interests: The authors state no conflict of interest. 
Informed consent: Informed consent was obtained from all individuals included in this study.

Ethical approval: Research involving human subjects complied with all relevant national regulations, institutional policies and was conducted in accordance with the tenets of the Helsinki Declaration (as revised in 2013). This research was approved by the Ethical Committee of the University of Leipzig (reference number: Reg. No. 264-10-19042010) and is registered in the clinical trials database (NCT02550236).

Data availability: The datasets generated during and/or analyzed during the mentioned sub-studies are available from the head of the LIFE child study and co-author of the manuscript, Prof. Wieland Kiess upon reasonable request.

\section{References}

1. Geserick M, Vogel M, Gausche R, Lipek T, Spielau U, Keller E, et al. Acceleration of BMI in early childhood and risk of sustained obesity. N Engl J Med 2018;379:1303-12.

2. Poulain T, Baber R, Vogel M, Pietzner D, Kirsten T, Jurkutat A, et al. LIFE Child study team. The LIFE Child study: a population-based perinatal and pediatric cohort in Germany. Eur J Epidemiol 2017; 32:145-58.

3. Surup H, Vogel M, Koerner A, Hiemisch A, Oelkers L, Willenberg A, et al. Pediatric reference intervals for thyrotropin, free triiodothyronine, and free thyroxine and the relevance of body mass index and puberty in measurement interpretation. Thyroid 2021;31:1192-202.

4. Eckelt F, Vogel M, Geserick M, Kirsten T, Bae YJ, Baber R, et al. Calcitonin measurement in pediatrics: reference ranges are gender-dependent, validation in medullary thyroid cancer and thyroid diseases. Clin Chem Lab Med 2019;57:1242-50.

5. Geserick M, Vogel M, Eckelt F, Schlingmann M, Hiemisch A, Baber $R$, et al. Children and adolescents with obesity have reduced serum bone turnover markers and 25 -hydroxyvitamin $D$ but increased parathyroid hormone concentrations - results derived from new pediatric reference ranges. Bone 2020;132:115124.

6. Bae YJ, Zeidler R, Baber R, Vogel M, Wirkner K, Loeffler M, et al. Reference intervals of nine steroid hormones over the life-span analyzed by LC-MS/MS: effect of age, gender, puberty, and oral contraceptives. J Steroid Biochem Mol Biol 2019;193:105409.

7. Gaudl A, Kratzsch J, Bae YJ, Kiess W, Thiery J, Ceglarek U. Liquid chromatography quadrupole linear ion trap mass spectrometry for quantitative steroid hormone analysis in plasma, urine, saliva and hair. J Chromatogr A 2016;1464:64-71.

8. Gaudl A, Kratzsch J, Ceglarek U. Advancement in steroid hormone analysis by LC-MS/MS in clinical routine diagnostics - a three year recap from serum cortisol to dried blood $17 \alpha$-hydroxyprogesterone. J Steroid Biochem Mol Biol 2019;192:105389.
9. Kromeyer-Hauschild K, Wabitsch M, Kunze D, Geller F, Geiß HC, Hesse V, et al. Perzentile für den Body-mass-Index für das Kindes- und Jugendalter unter Heranziehung verschiedener deutscher Stichproben. Monatsschr Kinderheilkd 2001;149: 807-18.

10. Marshall WA, Tanner JM. Variations in the pattern of pubertal changes in boys. Arch Dis Child 1970;45:13-23.

11. Marshall WA, Tanner JM. Variations in pattern of pubertal changes in girls. Arch Dis Child 1969;44:291-303.

12. Vogel M, Kirsten T, Kratzsch J, Engel C, Kiess W. A combined approach to generate laboratory reference intervals using unbalanced longitudinal data. J Pediatr Endocrinol Metab 2017; 30:767-73.

13. Sonntag J, Vogel M, Geserick M, Eckelt F, Körner A, Raue F, et al. Age-related association of calcitonin with parameters of anthropometry, bone and calcium metabolism during childhood. Horm Res Paediatr 2020;93:361-70.

14. Busch AS, Ljubicic ML, Upners EN, Fischer MB, Kolby N, EckertLind C, et al. Cohort profile: the COPENHAGEN Minipuberty Study-A longitudinal prospective cohort of healthy full-term infants and their parents. Paediatr Perinat Epidemiol 2021;35: 601-11.

15. Bidlingmaier M, Friedrich N, Emeny RT, Spranger J, Wolthers OD, Roswall J, et al. Reference intervals for insulin-like growth factor-1 (igf-i) from birth to senescence: results from a multicenter study using a new automated chemiluminescence IGF-I immunoassay conforming to recent international recommendations. J Clin Endocrinol Metab 2014;99:1712-21.

16. Friedrich N, Wolthers OD, Arafat AM, Emeny RT, Spranger J, Roswall J, et al. Age- and sex-specific reference intervals across life span for insulin-like growth factor binding protein 3 (IGFBP-3) and the IGF-I to IGFBP-3 ratio measured by new automated chemiluminescence assays. J Clin Endocrinol Metab 2014;99:1675-86.

17. Oron T, Lazar L, Feldhamer I, Manor M, Koren-Morag N, Tenenbaum A, et al. Pediatric reference values of TSH should be personalized according to BMI and ethnicity. Eur J Endocrinol 2020;183:419-26.

18. Schnabl K, Chan MK, Gong Y, Adeli K. Closing the gaps in paediatric reference intervals: the CALIPER initiative. Clin Biochem Rev 2008;29:89-96.

19. Zierk J, Krebs A, Rauh M, Metzler M, Löscher A, Strasser E, et al. Blood counts in adult and elderly individuals: defining the norms over eight decades of life. $\mathrm{Br} J$ Haematol 2020;189:777-89.

20. Zierk J, Baum H, Bertram A, Boeker M, Buchwald A, Cario H, et al. High-resolution pediatric reference intervals for 15 biochemical analytes described using fractional polynomials. Clin Chem Lab Med 2021;59:1267-78.

21. Argente Del Castillo P, Pastor García MI, Morell-Garcia D, Martinez-Gomez L, Ballesteros MA, Barcelo A. Thyroid panel reference intervals in healthy children and adolescents: $a$ Spanish cohort. Clin Biochem 2021;91:39-44.

22. Tate JR, Yen T, Jones GR. Transference and validation of reference intervals. Clin Chem 2015;61:1012-5. 